\title{
Amenability versus non amenability: an introduction to von Neumann algebras
}

\author{
Stefaan Vaes* \\ In European Congress of Mathematics, Berlin 2016, Eur. Math. Soc., \\ Zürich, 2018, pp. 483-500.
}

\begin{abstract}
The theory of von Neumann algebras was initiated by Murray and von Neumann and has deep connections to several areas of mathematics, in particular group theory and ergodic theory. Amenable von Neumann algebras were completely classified by Connes and Haagerup, while numerous classification theorems in the non amenable case were obtained within Popa's deformation/rigidity theory. This survey article provides an introduction to von Neumann algebras, written for non specialists and with the dichotomy between amenability and non amenability as our guide.
\end{abstract}

2010 Mathematics Subject Classification. Primary 46L36; Secondary 46L10, 28D15, 46L54, 20E22, 20E06.

Keywords. von Neumann algebras; deformation/rigidity theory; II $_{1}$ factors; type III factors; amenability.

\section{Banach-Tarski paradox, amenable groups, free groups}

In 1924, Banach and Tarski proved the "obviously false" theorem saying that a massive ball $B$ with radius 1 can be partitioned into finitely many subsets $B=$ $B_{1} \sqcup \cdots \sqcup B_{n}$ in such a way that two massive balls with radius 1 can be obtained by rotating and translating these pieces. Although called a paradox, this is a true theorem saying in particular that there is no finitely additive measure on $\mathbb{R}^{3}$ that is translation and rotation invariant and that gives a finite nonzero measure to the unit ball $B$.

In dimension two, the situation is drastically different. There do exist finitely additive, translation and rotation invariant measures on $\mathbb{R}^{2}$ that give measure 1 to the unit disc. So, there is no two dimensional Banach-Tarski paradox. A conceptual explanation was discovered by John von Neumann in [vN29] who proved that the group of motions of $\mathbb{R}^{3}$, viewed as a discrete group, is non amenable, while the group of motions of $\mathbb{R}^{2}$ is amenable.

More precisely, a discrete group $\Gamma$ is called amenable if there exists a finitely additive probability measure $m$ defined on all subsets of $\Gamma$ such that $m(g \mathcal{U})=m(\mathcal{U})$ for all $g \in \Gamma$ and $\mathcal{U} \subset \Gamma$. When an amenable group $\Gamma$ acts on a set $X$, the invariant

*Supported by ERC Consolidator Grant 614195, and by long term structural funding Methusalem grant of the Flemish Government. 
mean $m$ on $\Gamma$ can be used to construct a $\Gamma$-invariant mean on $X$. So, since the group of motions of $\mathbb{R}^{2}$ is amenable, there is no two dimensional Banach-Tarski paradox.

In dimension three, the rotation group $\mathrm{SO}(3)$ is non amenable. Two generically chosen rotations $a, b \in \mathrm{SO}(3)$ generate a copy of the so-called free group $\mathbb{F}_{2}$. We call reduced word any concatenation of the letters $a, a^{-1}, b, b^{-1}$ in which $a$ is never preceded or followed by $a^{-1}$ and $b$ is never preceded or followed by $b^{-1}$. Freeness of $a$ and $b$ means that a reduced word with the letters $a, a^{-1}, b, b^{-1}$ never defines the identity rotation. The free group $\mathbb{F}_{2}$ admits a paradoxical decomposition: denoting by $W(a) \subset \mathbb{F}_{2}$ the set of words that start with the letter $a$, and similarly defining $W\left(a^{-1}\right), W(b)$ and $W\left(b^{-1}\right)$, we find that

$$
\begin{aligned}
& \mathbb{F}_{2}=\{e\} \sqcup W(a) \sqcup W\left(a^{-1}\right) \sqcup W(b) \sqcup W\left(b^{-1}\right), \\
& \mathbb{F}_{2}=W(a) \sqcup a W\left(a^{-1}\right) \quad \text { and } \quad \mathbb{F}_{2}=W(b) \sqcup b W\left(b^{-1}\right) .
\end{aligned}
$$

It follows that $\mathbb{F}_{2}$ is non amenable. Whenever $\mathbb{F}_{2}$ acts freely on a set $X$, the above paradoxical decomposition of $\mathbb{F}_{2}$ can be transferred to a paradoxical decomposition of $X$. Of course, this does not literally apply to the action of $\mathbb{F}_{2}<\mathrm{SO}(3)$ on the unit ball of $\mathbb{R}^{3}$, but this is nevertheless the main idea to prove the Banach-Tarski theorem.

All finite groups and all abelian groups are amenable. Also, amenability passes to subgroups and direct limits, and is stable under extensions. One deduces that the group of motions of $\mathbb{R}^{2}$ is amenable. Beyond these basic properties, it can be very hard to decide whether a given group $\Gamma$ is amenable. Most notably, it is not known whether Thompson's group $F$ is amenable. This group can be defined as the group of piecewise linear bijections $f:[0,1] \rightarrow[0,1]$ with $f(0)=0, f(1)=1$, having all break points at dyadic rationals and all slopes being a power of 2 .

\section{Von Neumann algebras and amenability}

The dichotomy between amenability and non amenability is most notable in the theory of von Neumann algebras, introduced by Murray and von Neumann in [MvN36]. As we will see, von Neumann algebras arise naturally from groups and from actions of groups on measure spaces. In this way, they reflect some of the most intriguing analytic and ergodic theoretic properties of groups and group actions.

Given a Hilbert space $\mathcal{H}$ with scalar product $\langle\xi, \eta\rangle$, we denote by $B(\mathcal{H})$ the space of all bounded linear operators on $\mathcal{H}$. Every bounded operator $T \in B(\mathcal{H})$ has an Hermitian adjoint $T^{*} \in B(\mathcal{H})$ characterized by $\langle T \xi, \eta\rangle=\left\langle\xi, T^{*} \eta\right\rangle$. A net of operators $T_{k} \in B(\mathcal{H})$ is said to converge weakly to $T \in B(\mathcal{H})$ if $\lim _{k}\left\langle T_{k} \xi, \eta\right\rangle=$ $\langle T \xi, \eta\rangle$ for all $\xi, \eta \in \mathcal{H}$.

Definition 2.1. A von Neumann algebra is a weakly closed unital $*$-subalgebra of $B(\mathcal{H})$.

Whenever $M \subset B(\mathcal{H})$, we denote by $M^{\prime} \subset B(\mathcal{H})$ the commutant of $M$ defined as $M^{\prime}=\{T \in B(\mathcal{H}) \mid S T=T S$ for all $S \in M\}$. The fundamental bicommutant 
theorem of von Neumann says that a unital *-subalgebra $M \subset B(\mathcal{H})$ is weakly closed if and only if $M=M^{\prime \prime}$. This result illustrates very well a common theme in the theory of von Neumann algebras, where functional analytic aspects are very tightly connected to algebraic aspects of the theory. As another illustration, we mention that both the operator norm and the weak topology on the unit ball of $M$ are completely determined by the $*$-algebra structure of $M$, so that purely algebraic bijective $*$-isomorphisms between von Neumann algebras are automatically isometric and weakly continuous on the unit ball.

2.1. Group von Neumann algebras. Some of the easiest to define and at the same time, the least understood von Neumann algebras are those associated in $[\mathrm{MvN} 43]$ to a countable group $\Gamma$. Define the Hilbert space $\mathcal{H}=\ell^{2}(\Gamma)$ with its canonical orthonormal basis $\left(\delta_{g}\right)_{g \in \Gamma}$. The left regular representation $\lambda: \Gamma \rightarrow \mathcal{U}(\mathcal{H})$ is defined by $\lambda_{g} \delta_{h}=\delta_{g h}$ for all $g, h \in \Gamma$. The group von Neumann algebra $L(\Gamma)$ is the von Neumann algebra generated by the unitary operators $\lambda_{g}, g \in \Gamma$. As we will see below, many of the natural questions that one can ask about these group von Neumann algebras $L(\Gamma)$ are important open problems, the most tantalizing one being the free group factors isomorphism problem [Ka67]: is $L\left(\mathbb{F}_{n}\right) \cong L\left(\mathbb{F}_{m}\right)$ when $n \neq m$ and $n, m \geq 2$ ?

2.2. Factors of different types. Since von Neumann algebras are closed under Borel functional calculus, they contain plenty of projections (i.e. self adjoint idempotents). Therefore, a von Neumann algebra $M$ can be decomposed in a non trivial way as a direct sum $M \cong M_{1} \oplus M_{2}$ if and only if the center $\mathcal{Z}(M)$ of the algebra differs from $\mathbb{C} 1$. Von Neumann algebras with trivial center, $\mathcal{Z}(M)=\mathbb{C} 1$, are called factors. Every von Neumann algebra has a canonical decomposition as a generalized direct sum (called direct integral) of factors. The group von Neumann algebra $L(\Gamma)$ is a factor if and only if $\Gamma$ has infinite conjugacy classes (icc), meaning that $\left\{g h g^{-1} \mid g \in \Gamma\right\}$ is an infinite set for every $h \neq e$.

Group von Neumann algebras $M=L(\Gamma)$ are equipped with the canonical functional $\tau: M \rightarrow \mathbb{C}: \tau(x)=\left\langle x \delta_{e}, \delta_{e}\right\rangle$. One checks that $\tau(1)=1$ and $\tau\left(\lambda_{g}\right)=0$ for all $g \neq e$, so that $\tau\left(\lambda_{g} \lambda_{h}\right)=\tau\left(\lambda_{h} \lambda_{g}\right)$ and thus $\tau(x y)=\tau(y x)$ for all $x, y \in M$. We say that $\tau$ is a trace. Moreover, $\tau$ is a state $^{1}$ that is faithful ${ }^{2}$ and normal ${ }^{3}$. Factors admitting such a normal tracial state are said to be of type $I I_{1}$, except when they are finite dimensional, and thus isomorphic to $M_{n}(\mathbb{C})$, in which case they are said to be of type I.

A factor $M$ is said to be of type $I I_{\infty}$ when $M$ can be viewed as infinite matrices over a $\mathrm{II}_{1}$ factor. In more technical terms, this means that $M$ is isomorphic to the tensor product $M \cong N \bar{\otimes}(\mathcal{K})$ of a $\mathrm{II}_{1}$ factor $N$ and the bounded operators on an infinite dimensional Hilbert space $\mathcal{K}$. The factors that are not of type $\mathrm{I}_{1} \mathrm{II}_{1}$ or $\mathrm{II}_{\infty}$ are said to be of type III. This type classification was already developed in [MvN36].

\footnotetext{
${ }^{1} \mathrm{~A}$ state is a functional $\varphi: M \rightarrow \mathbb{C}$ such that $\varphi(1)=1$ and $\varphi\left(x^{*} x\right) \geq 0$ for all $x \in M$.

${ }^{2} \mathrm{~A}$ state $\varphi$ is called faithful if $\varphi\left(x^{*} x\right)>0$ for all $x \neq 0$.

${ }^{3} \mathrm{~A}$ functional is called normal if it is weakly continuous on the unit ball.
} 
Type $\mathrm{II}_{1}$ factors really are the basic building blocks of arbitrary von Neumann algebras. Above, we already mentioned the reduction to factors and the way to view $\mathrm{II}_{\infty}$ factors as infinite amplifications of $\mathrm{II}_{1}$ factors. When $M$ is an arbitrary von Neumann algebra and $\varphi: M \rightarrow \mathbb{C}$ is a faithful normal state, the trace property $\varphi(x y)=\varphi(y x)$ need not hold. However, by a fundamental discovery of Tomita and Takesaki [Ta70], the trace property can be replaced by the formula $\varphi(x y)=\varphi\left(\sigma_{\mathrm{i}}^{\varphi}(y) x\right)$. Here $\left(\sigma_{t}^{\varphi}\right)_{t \in \mathbb{R}}$ is a canonical 1-parameter group of automorphisms of $M$, called the modular automorphism group of $\varphi$, and $\sigma_{i}^{\varphi}(y)$ is its analytic continuation, which is only well defined on a canonical dense subalgebra of $M$. By an equally striking discovery of Connes [Co72], the modular automorphism group $\left(\sigma_{t}^{\varphi}\right)_{t \in \mathbb{R}}$ is essentially independent of the choice of the state $\varphi$. This allowed Connes and Takesaki in [Co72, Ta73] to canonically decompose a type III von Neumann algebra as a crossed product of a von Neumann algebra $N$ of type $\mathrm{II}_{\infty}$ and a trace scaling 1-parameter group of automorphisms of $N$. Depending on how large the center of $N$ is, type III factors can then be further classified into factors of type $\mathrm{III}_{\lambda}$ with $\lambda \in[0,1]$, see [Co72].

2.3. The hyperfinite $\mathbf{I I}_{1}$ factor. Define $A_{k}=M_{2^{k}}(\mathbb{C})$ and view $A_{k} \hookrightarrow A_{k+1}$ by $x \mapsto\left(\begin{array}{cc}x & 0 \\ 0 & x\end{array}\right)$. We equip $A_{k}$ with the normalized trace $\tau=2^{-k} \operatorname{Tr}$. These traces are compatible with the inclusions. Passing to the direct limit, we find a *-algebra $\mathcal{A}$ and a positive tracial functional $\tau: \mathcal{A} \rightarrow \mathbb{C}$. Using the inner product $\langle x, y\rangle=\tau\left(x y^{*}\right)$, we complete $\mathcal{A}$ to a Hilbert space $\mathcal{H}$ on which $\mathcal{A}$ is represented by left multiplication operators. The weak closure of $\mathcal{A}$ inside $B(\mathcal{H})$ is the hyperfinite $I I_{1}$ factor $R$ of Murray and von Neumann [MvN43]. This is the most basic example of a $\mathrm{II}_{1}$ factor, in particular because every $\mathrm{II}_{1}$ factor $M$ contains many copies of $R$ as a von Neumann subalgebra $R \subset M$.

In general, a factor $M$ is said to be hyperfinite if it contains an increasing sequence of finite dimensional *-subalgebras $A_{k} \subset M$ such that $\bigcup_{k} A_{k}$ is weakly dense in $M$. Already Murray and von Neumann proved in [MvN43] the striking classification theorem saying that all hyperfinite $\mathrm{II}_{1}$ factors are isomorphic.

2.4. Connes' classification of amenable factors. A von Neumann algebra $M \subset B(\mathcal{H})$ is said to be amenable if the correct analog of an invariant mean exists: a linear map $E: B(\mathcal{H}) \rightarrow M$ that is positive, in the sense that $E(T) \geq 0$ for every $T \geq 0$, unital and that satisfies $E(x T y)=x E(T) y$ for all $T \in B(\mathcal{H})$ and $x, y \in M$. We say that $E$ is a conditional expectation of $B(\mathcal{H})$ onto $M$. Note that we do not assume any weak continuity for $E$.

One checks that a group von Neumann algebra $L(\Gamma)$ is amenable if and only if the group $\Gamma$ is amenable. So, for all amenable icc groups $\Gamma$, we get that $L(\Gamma)$ is an amenable $\mathrm{II}_{1}$ factor. The following theorem of Connes is thus a big surprise.

Theorem 2.2 (Connes [Co75]). All amenable $I_{1}$ factors are hyperfinite and thus, isomorphic with the hyperfinite $I_{1}$ factor $R$ of Murray and von Neumann.

In [Co75], Connes proved the general result that all amenable factors acting on a separable Hilbert space are hyperfinite. Using the Tomita-Takesaki-Connes 
decomposition theory discussed above, Connes could then show that for every $\lambda \in(0,1)$, there is a unique amenable factor of type $\mathrm{III}_{\lambda}$ and that the amenable factors of type $\mathrm{III}_{0}$ are completely classified by an ergodic, non transitive flow. The uniqueness of the hyperfinite $\mathrm{III}_{1}$ factor remained open and was ultimately proved by Haagerup in [Ha85].

For $\lambda \in(0,1)$, the unique amenable factor of type $\mathrm{III}_{\lambda}$ is the Powers factor $R_{\lambda}$, which can be constructed as follows. Consider the same inclusions $A_{k} \hookrightarrow A_{k+1}$ of $A_{k}=M_{2^{k}}(\mathbb{C})$ as in Section 2.3. Inductively define the states $\varphi_{k}$ on $A_{k}$ given by

$$
\varphi_{k+1}\left(\begin{array}{ll}
a & b \\
c & d
\end{array}\right)=\frac{1}{1+\lambda} \varphi_{k}(a)+\frac{\lambda}{1+\lambda} \varphi_{k}(d) \quad \text { for all } a, b, c, d \in A_{k} .
$$

Denote by $\mathcal{A}$ the inductive limit $*$-algebra with the inductive limit state $\varphi$. We complete $\mathcal{A}$ into a Hilbert space $\mathcal{H}$ by using the scalar product $\langle a, b\rangle=\varphi\left(b^{*} a\right)$. Again, left multiplication defines a representation of $\mathcal{A}$ on $\mathcal{H}$ and $R_{\lambda}$ is defined as the weak closure of $\mathcal{A} \subset B(\mathcal{H})$.

\section{Popa's deformation/rigidity theory and non amenability}

Rigidity phenomena coming from non amenability already appeared early in the development of von Neumann algebras, notably in proving that the free group factors $L\left(\mathbb{F}_{n}\right)$ are not isomorphic to the hyperfinite $\mathrm{II}_{1}$ factor [MvN43], or in constructing uncountably many non isomorphic $\mathrm{II}_{1}$ factors [Mc69]. Nevertheless, most of the natural isomorphism and classification problems for families of non amenable $\mathrm{II}_{1}$ factors remained wide open. This changed dramatically in the early 2000's, when Popa introduced his deformation/rigidity theory in [Po01]. Since the developments in deformation/rigidity theory in the period 2001-2012 have been surveyed in [Po06, Va10, Io12a], I focus here on a sample of results that illustrate best the dichotomy between amenability and non amenability, as well as on a few of the most recent developments.

3.1. Group measure space $\mathbf{I I}_{\mathbf{1}}$ factors. Let $(P, \tau)$ be a von Neumann algebra equipped with a faithful normal tracial state and let $\Gamma \curvearrowright^{\alpha}(P, \tau)$ be an action of a countable group $\Gamma$ by trace preserving automorphisms $\left(\alpha_{g}\right)_{g \in \Gamma}$ of $(P, \tau)$. Most of the time, we will take $P=L^{\infty}(X)$, where $(X, \mu)$ is a standard probability space, $\tau(F)=\int_{X} F(x) d \mu(x)$ and $\Gamma \curvearrowright^{\alpha} L^{\infty}(X)$ is induced from a probability measure preserving (pmp) action $\Gamma \curvearrowright(X, \mu)$ via $\left(\alpha_{g}(F)\right)(x)=F\left(g^{-1} \cdot x\right)$.

The crossed product $M=P \rtimes \Gamma$ is the unique von Neumann algebra with faithful normal tracial state $\tau: M \rightarrow \mathbb{C}$ satisfying the following.

- $M$ contains $P$ as a unital *-subalgebra and contains a copy of $\Gamma$ as unitary operators $\left(u_{g}\right)_{g \in \Gamma}$.

- We have $u_{g} u_{h}=u_{g h}$ and $u_{g} x u_{g}^{*}=\alpha_{g}(x)$ for all $g, h \in \Gamma, x \in P$.

- The finite sums $\sum_{g \in \Gamma} x_{g} u_{g}$ with $x_{g} \in P$ form a weakly dense $*$-subalgebra of $M$. 
- We have $\tau\left(\sum_{g \in \Gamma} x_{g} u_{g}\right)=\tau\left(x_{e}\right)$.

For a pmp action $\Gamma \curvearrowright(X, \mu)$, two natural properties emerge. The abelian subalgebra $L^{\infty}(X) \subset L^{\infty}(X) \rtimes \Gamma$ is maximal abelian if and only if the action $\Gamma \curvearrowright(X, \mu)$ is essentially free, meaning that $\{x \in X \mid g \cdot x=x\}$ has measure zero for every $g \neq e$. Under this freeness assumption, we find that $L^{\infty}(X) \rtimes \Gamma$ is a factor if and only if the action $\Gamma \curvearrowright(X, \mu)$ is ergodic, meaning that the only $\Gamma$-invariant functions in $L^{\infty}(X)$ are the constant functions. So, for all free ergodic pmp actions $\Gamma \curvearrowright(X, \mu)$, we conclude that $L^{\infty}(X) \rtimes \Gamma$ is a $\mathrm{II}_{1}$ factor. This is the group measure space construction of $[\mathrm{MvN} 36]$.

The group measure construction $L^{\infty}(X) \rtimes \Gamma$ can be defined for non singular actions $\Gamma \curvearrowright(X, \mu)$, meaning that $\mu(g \cdot \mathcal{U})=0$ for all Borel sets $\mathcal{U} \subset X$ with $\mu(\mathcal{U})=0$ and all $g \in \Gamma$. For a free, ergodic, non singular action $\Gamma \curvearrowright(X, \mu)$, we still get that $M=L^{\infty}(X) \rtimes \Gamma$ is a factor. The type of $M$ is determined as follows by the dynamics of the action: $M$ is of type I if and only if $\mu$ is concentrated on a single orbit; $M$ is of type $\mathrm{II}_{1}$ if and only if there exists a $\Gamma$-invariant, non atomic probability measure in the same measure class as $\mu$ (which is the case discussed above); $M$ is of type $\mathrm{II}_{\infty}$ if and only if there exists an infinite, $\sigma$-finite, $\Gamma$-invariant, non atomic measure in the same measure class as $\mu$; and in all other cases, $M$ is of type III.

3.2. Classifying group measure space $\mathrm{II}_{\mathbf{1}}$ factors. The basic question is: when are two given group measure space $\mathrm{II}_{1}$ factors isomorphic? By Connes' Theorem 2.2, for all free ergodic pmp actions of an amenable group $\Gamma$, we have $L^{\infty}(X) \rtimes \Gamma \cong R$. But what happens for concrete actions of various natural groups such as the free groups $\mathbb{F}_{n}$ or the groups $\mathrm{SL}(n, \mathbb{Z})$ ? Until the advent of Popa's deformation/rigidity theory, most of such questions were out of reach.

Assume that $\pi: L^{\infty}(X) \rtimes \Gamma \rightarrow L^{\infty}(Y) \rtimes \Lambda$ is an isomorphism. In [Po01], Popa proved that if $\Lambda$ has the Haagerup property (a deformation property that I introduce below), while $\Gamma \curvearrowright(X, \mu)$ is a rigid action (meaning that $L^{\infty}(X)$ is a subalgebra having the relative property $(\mathrm{T})$ ), then there must exist a unitary operator $u$ such that the new isomorphism $(\operatorname{Ad} u) \circ \pi$ maps $L^{\infty}(X)$ onto $L^{\infty}(Y)$. By Singer's theorem, this implies that the actions $\Gamma \curvearrowright(X, \mu)$ and $\Lambda \curvearrowright(Y, \nu)$ are orbit equivalent, meaning that there exists a measure preserving Borel bijection $\Delta: X \rightarrow Y$ such that $\Delta(\Gamma \cdot x)=\Lambda \cdot \Delta(x)$ for almost every $x \in X$.

So, Popa's theorem reduced for the first time in a specific setting the isomorphism problem of group measure space $\mathrm{II}_{1}$ factors to the isomorphism problem of the associated orbit equivalence relations. That is a question in measured group theory that we do not discuss further here, see [Ga10, Fu11]. We mention however that the $L^{2}$-Betti numbers of a countable group $\Gamma$ are preserved under orbit equivalence (see [Ga01]) and thus become an invariant for the class of $\mathrm{II}_{1}$ factors introduced by Popa in [Po01].

A countable group $\Lambda$ has the Haagerup approximation property if there exists a sequence of positive definite functions $\varphi_{n}: \Lambda \rightarrow \mathbb{C}$ converging pointwise to 1 and with each $\varphi_{n}$ being a function that tends to 0 at infinity. This approximation of the identity induces on a crossed product $M=P \rtimes \Lambda$ a sequence of normal 
completely positive maps given by

$$
\psi_{n}: M \rightarrow M: \psi_{n}\left(\sum_{g} x_{g} u_{g}\right)=\sum_{g} \varphi_{n}(g) x_{g} u_{g}
$$

satisfying $\lim _{n}\left\|\psi_{n}(x)-x\right\|_{2}=0$ for every $x \in M$, where $\|x\|_{2}=\sqrt{\tau\left(x^{*} x\right)}$. A von Neumann subalgebra $Q \subset M$ is called rigid [Po01] (or has the relative property $(T)$ ) if for any sequence of normal completely positive maps $\psi_{n}: M \rightarrow M$ converging pointwise to the identity, we have that $\left\|\psi_{n}(x)-x\right\|_{2}$ tends to zero uniformly on all $x$ in the unit ball of $Q$. In a setting as in the previous paragraphs, having both deformation and rigidity, one may uniquely localize the rigid subalgebra and then use this as the crucial step in proving a classification theorem.

3.3. Bernoulli actions and $\mathbf{W}^{*}$-superrigidity. The Bernoulli action of a countable group $\Gamma$ with base space $\left(X_{0}, \mu_{0}\right)$ is defined as

$$
\Gamma \curvearrowright(X, \mu)=\left(X_{0}, \mu_{0}\right)^{\Gamma}:(g \cdot x)_{h}=x_{g^{-1} h} .
$$

Some of the most striking rigidity phenomena in von Neumann algebras have been found for Bernoulli actions of non amenable groups. They all originate from the following result and the methods developed in [Po03, Po04].

Theorem 3.1 (Popa, [Po03, Po04]). Let $\Gamma \curvearrowright(X, \mu)$ and $\Lambda \curvearrowright(Y, \nu)$ be free ergodic pmp actions of icc groups $\Gamma$ and $\Lambda$. Assume that $\Gamma$ has Kazhdan's property $(T)$ and that $\Lambda \curvearrowright(Y, \nu)$ is a Bernoulli action. If the crossed products are isomorphic, i.e. $L^{\infty}(X) \rtimes \Gamma \cong L^{\infty}(Y) \rtimes \Lambda$, then $\Gamma \cong \Lambda$ and the actions $\Gamma \curvearrowright(X, \mu)$ and $\Lambda \curvearrowright(Y, \nu)$ are conjugate.

Recall here that free ergodic pmp actions $\Gamma \curvearrowright(X, \mu)$ and $\Lambda \curvearrowright(Y, \nu)$ are called conjugate if there exists an isomorphism of groups $\delta: \Gamma \rightarrow \Lambda$ and a measure preserving Borel bijection $\Delta: X \rightarrow Y$ such that $\Delta(g \cdot x)=\delta(g) \cdot \Delta(x)$ for all $g \in \Gamma$ and almost every $x \in X$. Similarly, these actions are called $W^{*}$-equivalent if the associated group measure space $\mathrm{II}_{1}$ factors are isomorphic: $L^{\infty}(X) \rtimes \Gamma \cong$ $L^{\infty}(Y) \rtimes \Lambda$.

The rigidity in Theorem 3.1 is given by property $(\mathrm{T})$ groups such as $\mathrm{SL}(n, \mathbb{Z})$, $n \geq 3$, or, more generally, lattices in higher rank simple Lie groups. The deformation in Theorem 3.1 is given by Popa's malleability property of Bernoulli actions $\Lambda \curvearrowright(Y, \nu)$ : there exists a 1-parameter group of pmp transformations $\alpha_{t}: Y \times Y \rightarrow$ $Y \times Y$ such that $\alpha_{t}$ commutes with the diagonal action $g \cdot(x, y)=(g \cdot x, g \cdot y)$ and such that $\alpha_{0}=$ id and $\alpha_{1}(x, y)=(y, x)$.

Note that the hypotheses in Theorem 3.1 are asymmetric: there is an assumption on the group $\Gamma$, while there is an assumption on the action of $\Lambda$. The most extreme form of rigidity arises when the same conclusion holds by only making assumptions on one side. This leads us to the following concept.

Definition 3.2. A free ergodic pmp action $\Gamma \curvearrowright(X, \mu)$ is said to be $W^{*}$-superrigid if any free ergodic pmp action that is $\mathrm{W}^{*}$-equivalent, must be conjugate. 
In [Pe09], Peterson proved the existence of virtually $\mathrm{W}^{*}$-superrigid actions. Here, virtual means that the conjugacy in the conclusion only holds after restricting to finite index subgroups. Shortly afterwards, Popa and I proved in [PV09] that for large classes of amalgamated free product groups, the Bernoulli actions are $\mathrm{W}^{*}$-superrigid, while in [Io10], Ioana proved the same for property (T) groups.

Theorem 3.3. When $\Gamma$ is one of the following groups, any Bernoulli action $\Gamma \curvearrowright$ $\left(X_{0}, \mu_{0}\right)^{\Gamma}$ is $W^{*}$-superrigid.

- (Popa-V, [PV09]) $\Gamma$ is an amalgamated free product group of the form $\Gamma=$ $\operatorname{PSL}(n, \mathbb{Z}) *_{T_{n}}\left(T_{n} \times \Lambda\right)$, where $n \geq 3, T_{n}<\operatorname{PSL}(n, \mathbb{Z})$ is the subgroup of upper triangular matrices and $\Lambda$ is an arbitrary non trivial group.

- (Ioana, [Io10]) $\Gamma$ is an infinite property (T) group.

The method to prove Theorem 3.3 starts as follows ${ }^{4}$. Put $M=L^{\infty}(X) \rtimes \Gamma$ and assume that $M=B \rtimes \Lambda$ with $B=L^{\infty}(Y)$ is another group measure space decomposition of $M$. This "mysterious" group measure space decomposition of $M$ induces the injective $*$-homomorphism

$$
\Delta: M \rightarrow M \bar{\otimes} M: \Delta\left(\sum_{s \in \Lambda} b_{s} v_{s}\right)=\sum_{s \in \Lambda} b_{s} v_{s} \otimes v_{s}
$$

called the dual coaction. Using now the structure of $M$ as a Bernoulli crossed product by a specific type of group, we can partially unravel how such an embedding $\Delta: M \rightarrow M \bar{\otimes} M$ may look like. This ultimately allows to prove that $L^{\infty}(X)$ and $B$ are unitarily conjugate in $M$. As discussed above, it follows that the actions $\Gamma \curvearrowright(X, \mu)$ and $\Lambda \curvearrowright(Y, \nu)$ are orbit equivalent. Using Popa's orbit equivalence superrigidity theorem of [Po05], it follows that the actions must be conjugate.

3.4. $W^{*}$-superrigidity for group von Neumann algebras. How much information on a group $\Gamma$ can be recovered from the ambient group von Neumann algebra $L(\Gamma)$ ? Several qualitative properties of the group $\Gamma$ turned out to actually be properties of $L(\Gamma)$. This includes amenability, the Haagerup property, property $(\mathrm{T})$, etc. The more precise question when given groups $\Gamma$ and $\Lambda$ give rise to isomorphic group von Neumann algebras $L(\Gamma) \cong L(\Lambda)$ remains wide open for most natural families of groups.

We already mentioned the free group factor problem asking whether $L\left(\mathbb{F}_{n}\right)$ is isomorphic with $L\left(\mathbb{F}_{m}\right)$ when $n, m \geq 2$ and $n \neq m$. Equally wide open is Connes' rigidity conjecture saying that lattices in higher rank simple Lie groups, or even all property $(\mathrm{T})$ groups are $W^{*}$-superrigid in the following sense.

Definition 3.4. A countable group $\Gamma$ is said to be $\mathrm{W}^{*}$-superrigid if any group $\Lambda$ with $L(\Gamma) \cong L(\Lambda)$ satisfies $\Gamma \cong \Lambda$.

\footnotetext{
${ }^{4}$ The method as we describe it here was developed in [Io10], but the dual coaction in (3.1) was already a crucial ingredient in [PV09].
} 
In [IPV10], the first $\mathrm{W}^{*}$-superrigidity theorem for group von Neumann algebras was proved, for groups $G$ of the following form. Starting with an action of a countable group $\Gamma$ on a countable set $I$ (typically, $I=\Gamma / \Gamma_{0}$ for a subgroup $\Gamma_{0}<\Gamma$ ), define $H:=(\mathbb{Z} / 2 \mathbb{Z})^{(I)}$ as the direct sum of copies of the group with two elements and let $\Gamma$ act by automorphisms of $H$ given by the (generalized) Bernoulli action: $(g \cdot x)_{i}=x_{g^{-1} \cdot i}$ for all $g \in \Gamma, x \in H, i \in I$. Then define $G$ as the semidirect product

$$
G=\left(\frac{\mathbb{Z}}{2 \mathbb{Z}}\right)^{(I)} \rtimes \Gamma
$$

In [IPV10], we proved that for well chosen $\Gamma \curvearrowright I$, this group $G$ is $\mathrm{W}^{*}$-superrigid.

The method to prove this results starts again as follows. Given another decomposition $L(G)=L(\Lambda)$ as a group von Neumann algebra, we consider the injective *-homomorphism $\Delta: L(\Lambda) \rightarrow L(\Lambda) \bar{\otimes} L(\Lambda)$ given by $\Delta\left(v_{s}\right)=v_{s} \otimes v_{s}$ for all $s \in \Lambda$ and called the comultiplication on $L(\Lambda)$. Viewing $L(G)$ as the generalized Bernoulli crossed product

$$
L(G)=L^{\infty}\left(\{0,1\}^{I}\right) \rtimes \Gamma,
$$

we can determine how $\Delta$ must look like and then prove that $\Lambda \cong G$.

In [BV14], these methods were further refined and we could prove that for the left-right translation action $\Gamma \times \Gamma \curvearrowright \Gamma$ of the free groups $\Gamma=\mathbb{F}_{n}, n \geq 2$, the semidirect product group $(\mathbb{Z} / 2 \mathbb{Z})^{(\Gamma)} \rtimes(\Gamma \times \Gamma)$ is $\mathrm{W}^{*}$-superrigid.

3.5. Uniqueness of Cartan subalgebras. As explained in Section 3.3, proving $\mathrm{W}^{*}$-superrigidity of a free ergodic pmp action $\Gamma \curvearrowright(X, \mu)$ proceeds in two steps. Write $A=L^{\infty}(X)$ and $M=A \rtimes \Gamma$. If $M=B \rtimes \Lambda$ is another group measure space decomposition of $M$, induced by a free ergodic pmp action $\Lambda \curvearrowright(Y, \nu)$ and writing $B=L^{\infty}(Y)$, the first step consists in proving that $A$ and $B$ are unitarily conjugate subalgebras of $M$, i.e. there exists a unitary operator $u \in \mathcal{U}(M)$ such that $B=u A u^{*}$. It then follows that the actions $\Gamma \curvearrowright(X, \mu)$ and $\Lambda \curvearrowright(Y, \nu)$ are orbit equivalent, so that the second step consists in proving the orbit equivalence superrigidity of $\Gamma \curvearrowright(X, \mu)$.

The subalgebra $A \subset M$ is a Cartan subalgebra: a maximal abelian subalgebra whose normalizer

$$
\mathcal{N}_{M}(A)=\left\{u \in \mathcal{U}(M) \mid u A u^{*}=A\right\}
$$

generates $M$ as a von Neumann algebra. When a Cartan subalgebra $A \subset M$ arises from a group measure space decomposition $M=L^{\infty}(X) \rtimes \Gamma$, where $\Gamma \curvearrowright$ $(X, \mu)$ is a free ergodic pmp action, we call $A$ a group measure space (gms) Cartan subalgebra. Not all Cartan subalgebras of a $\mathrm{II}_{1}$ factor are of group measure space type. Indeed, by Feldman and Moore's theorem [FM75], a Cartan inclusion $A \subset M$ is always of the form $L^{\infty}(X) \subset L_{\Omega}(\mathcal{R})$, where $(X, \mu)$ is a standard probability space, $\mathcal{R}$ is a Borel equivalence relation on $X$ with countable equivalence classes, $\mathcal{R}$ is "preserving" the probability measure $\mu$ and $\Omega$ is a 2-cocycle on $\mathcal{R}$. There are two gaps between this general setup and a group measure space decomposition. The first issue is that $\mathcal{R}$ need not be the orbit equivalence relation $\mathcal{R}=\{(x, g \cdot x) \mid$ 
$x \in X, g \in \Gamma\}$ of a free pmp action $\Gamma \curvearrowright(X, \mu)$. Secondly, even if $\mathcal{R}$ is such an orbit equivalence relation, the 2-cocycle $\Omega$ can be non trivial.

The first step in proving $\mathrm{W}^{*}$-superrigidity can now be formulated as follows: is $L^{\infty}(X)$ the unique gms Cartan subalgebra of $L^{\infty}(X) \rtimes \Gamma$ up to unitary conjugacy? Clearly, the more natural question to ask is whether $L^{\infty}(X)$ simply is the unique Cartan subalgebra up to unitary conjugacy. That type of question turned out to be much harder to solve because a dual coaction as in (3.1) is no longer available.

Only in [OP07], the first $\mathrm{II}_{1}$ factor with a unique Cartan subalgebra up to unitary conjugacy was discovered. They proved that whenever $\Gamma=\mathbb{F}_{n}$ is a free group with $n \geq 2$ and $\Gamma_{k}<\Gamma$ is a decreasing sequence of finite index normal subgroups with $\bigcap_{k} \Gamma_{k}=\{e\}$, then the profinite action $\Gamma \curvearrowright X=\lim \Gamma / \Gamma_{k}$ gives rise to a $\mathrm{II}_{1}$ factor $M=L^{\infty}(X) \rtimes \Gamma$ in which $L^{\infty}(X)$ is the unique Cartan subalgebra up to unitary conjugacy. In [CS11], this uniqueness theorem was generalized to $\mathrm{II}_{1}$ factors given by profinite actions of arbitrary non elementary hyperbolic groups.

In both cases, the crucial ingredient is the weak amenability of profinite crossed products $M=L^{\infty}(X) \rtimes \Gamma$ by a weakly amenable group $\Gamma$. This provides a sequence of finite rank, normal, completely bounded maps $\psi_{n}: M \rightarrow M$ such that $\lim _{n}\left\|\psi_{n}(x)-x\right\|_{2}=0$ for all $x \in M$ and such that $\lim \sup _{n}\left\|\psi_{n}\right\|_{\mathrm{cb}}<\infty$. Here, $\|\psi\|_{\mathrm{cb}}$ is defined as the supremum of all norms $\|$ id $\otimes \psi: M_{k}(\mathbb{C}) \otimes M \rightarrow M_{k}(\mathbb{C}) \otimes M \|$, $k \in \mathbb{N}$, and a map $\psi$ is called completely bounded if $\|\psi\|_{\mathrm{cb}}<\infty$. Whenever $B \subset M$ is an amenable von Neumann subalgebra (e.g. another Cartan subalgebra), the formula $\mu_{n}\left(b \otimes c^{\mathrm{op}}\right)=\tau\left(\psi_{n}(b) c\right)$ gives rise to a sequence of normal functionals $\mu_{n}$ on $B \bar{\otimes} B^{\text {op }}$ satisfying $\lim \sup _{n}\left\|\mu_{n}\right\|<\infty$. This sequence $\mu_{n}$ turns out to have remarkable asymptotic invariance properties under a natural action of the normalizer $\mathcal{N}_{M}(B)$. In this way, it is proved in [OP07] that whenever $B$ is amenable and diffuse $^{5}$, then the normalizer $\mathcal{N}_{M}(B)^{\prime \prime}$ stays amenable.

When $\Gamma$ is still weakly amenable but $\Gamma \curvearrowright(X, \mu)$ is an arbitrary free ergodic pmp action, the crossed product $L^{\infty}(X) \rtimes \Gamma$ need not be weakly amenable (this is the case whenever $\Gamma$ is non amenable and $\Gamma \curvearrowright(X, \mu)$ is a Bernoulli action [OP07]). Nevertheless in [PV11, PV12], we construct a natural von Neumann algebra that replaces $B \bar{\otimes} B^{\text {op }}$ and that admits a sequence of normal functionals $\mu_{n}$ with the appropriate asymptotic invariance properties. This then leads to the following result.

Theorem 3.5 (Popa-V, [PV11, PV12]). Let $\Gamma$ be the free group $\mathbb{F}_{n}, n \geq 2$, or any non elementary hyperbolic group. For any free ergodic pmp action $\Gamma \curvearrowright(X, \mu)$, we have that $L^{\infty}(X)$ is the unique Cartan subalgebra of $L^{\infty}(X) \rtimes \Gamma$ up to unitary conjugacy.

Rather than directly proving Theorem 3.5, we prove in [PV11, PV12] a general dichotomy theorem for arbitrary tracial crossed products $M=P \rtimes \Gamma$ and von Neumann subalgebras $Q \subset M$ that are amenable relative to $P$. This theorem says that either $Q$ can essentially be unitarily conjugated into $P$ or the normalizer

\footnotetext{
${ }^{5} \mathrm{~A}$ von Neumann algebra $B$ is called diffuse if it has no minimal projections. This is equivalent to saying that $B$ cannot be written as $B \cong B(\mathcal{K}) \oplus B_{1}$ for some finite or infinite dimensional Hilbert space $\mathcal{K}$.
} 
$\mathcal{N}_{M}(Q)^{\prime \prime}$ stays amenable relative to $P$. I do not elaborate this further here, but it is important to note that this general result has lead to further uniqueness theorems for Cartan subalgebras, in particular Ioana's theorem [Io12b] saying that uniqueness holds for arbitrary crossed products of free product groups $\Gamma=\Gamma_{1} * \Gamma_{2}$ with $\left|\Gamma_{1}\right| \geq 3$ and $\left|\Gamma_{2}\right| \geq 2$. The starting point of [Io12b] is that any crossed product $M=L^{\infty}(X) \rtimes \Gamma$ by such a free product group $\Gamma$ admits a family of embeddings

$$
\theta_{t}: M \hookrightarrow L^{\infty}(X) \rtimes\left(\left(\Gamma_{1} * \mathbb{Z}\right) *\left(\Gamma_{2} * \mathbb{Z}\right)\right) \cong P \rtimes \mathbb{F}_{2}
$$

so that the general dichotomy theorem of [PV11] can be applied to $\theta_{t}\left(L^{\infty}(X)\right) \subset$ $P \rtimes \mathbb{F}_{2}$.

Note that combining Theorem 3.5 with the invariance of $L^{2}$-Betti numbers under orbit equivalence (see [Ga01]), we obtain the following.

Corollary 3.6 ([PV11]). If $\mathbb{F}_{n} \curvearrowright(X, \mu)$ and $\mathbb{F}_{m} \curvearrowright(Y, \nu)$ are free ergodic pmp actions and $L^{\infty}(X) \rtimes \mathbb{F}_{n} \cong L^{\infty}(Y) \rtimes \mathbb{F}_{m}$, then $n=m$.

3.6. $\mathrm{II}_{1}$ factors with exactly two group measure space decompositions. Crossed product $\mathrm{II}_{1}$ factors $M$ need not have a unique Cartan subalgebra, not even up to conjugacy by an automorphism of $M$. This can for instance be seen in the following example of Ozawa and Popa. Consider the semidirect product group $\Gamma=\mathbb{Z}^{2} \rtimes \mathrm{SL}(2, \mathbb{Z})$ and the probability space $\mathbb{Z}_{p}^{2}$, where $\mathbb{Z}_{p}$ is the compact group of $p$-adic integers equipped with its Haar probability measure. The translation action $\mathbb{Z}^{2} \curvearrowright \mathbb{Z}_{p}^{2}$ and the "linear" action $\operatorname{SL}(2, \mathbb{Z}) \curvearrowright \mathbb{Z}_{p}^{2}$ combine into a free ergodic pmp action $\Gamma \curvearrowright \mathbb{Z}_{p}^{2}$. Inside the crossed product

$$
M=L^{\infty}\left(\mathbb{Z}_{p}^{2}\right) \rtimes\left(\mathbb{Z}^{2} \rtimes \mathrm{SL}(2, \mathbb{Z})\right),
$$

we not only have the canonical Cartan subalgebra $L^{\infty}\left(\mathbb{Z}_{p}^{2}\right)$, but also the group von Neumann algebra $L\left(\mathbb{Z}^{2}\right) \subset M$ is a Cartan subalgebra. These Cartan subalgebras are not conjugate by an automorphism of $M$.

It is an open problem to decide whether $L^{\infty}\left(\mathbb{Z}_{p}^{2}\right)$ and $L\left(\mathbb{Z}^{2}\right)$ are the only Cartan subalgebras of $M$ up to unitary conjugacy. Actually, it is an open problem to construct any $\mathrm{II}_{1}$ factor with precisely two Cartan subalgebras up to unitary conjugacy. Even more, it is an open problem to construct a $\mathrm{II}_{1}$ factor $M$ with more than one Cartan subalgebra but such that all these Cartan subalgebras can be exhaustively determined.

Nevertheless in [KV15], we could solve these questions when restricting to group measure space (gms) Cartan subalgebras.

Theorem 3.7 (Krogager-V, [KV15]). For every integer $n \geq 1$, we construct

- $I I_{1}$ factors $M$ having exactly $n$ gms Cartan subalgebras up to conjugacy by an automorphism of $M$,

- $I I_{1}$ factors $M$ having exactly $2^{n}$ gms Cartan subalgebras up to unitary conjugacy. 
The $\mathrm{II}_{1}$ factors $M$ in Theorem 3.7 are constructed as follows. Denote by $\left(R_{0}, \tau_{0}\right)$ the hyperfinite $\mathrm{II}_{1}$ factor with its tracial state. Let $\Gamma=\mathbb{F}_{\infty}$ and denote by $(R, \tau)$ the infinite tensor product $(R, \tau)=\bar{\otimes}_{g \in \Gamma}\left(R_{0}, \tau_{0}\right)$. For every $k \in \Gamma$, we denote by $\pi_{k}: R_{0} \rightarrow R$ the embedding as the $k$ 'th tensor factor. Given any trace preserving action $\Gamma \curvearrowright^{\beta} R_{0}$, we define the action $\Gamma \times \Gamma \curvearrowright^{\alpha} R$ given by

$$
\alpha_{(g, h)}\left(\pi_{k}(x)\right)=\pi_{g k h^{-1}}\left(\beta_{h}(x)\right)
$$

We define $M$ as the crossed product $\mathrm{II}_{1}$ factor $M=R \rtimes(\Gamma \times \Gamma)$. The obvious Cartan subalgebras $B \subset M$ are the ones of the form $B=B_{0}^{\bar{\otimes} \Gamma}$ where $B_{0} \subset R_{0}$ is a Cartan subalgebra that is invariant under $\beta$, meaning that $\beta_{h}\left(B_{0}\right)=B_{0}$ for all $h \in \Gamma$. The obvious gms decompositions of $M$ are the ones of the form

$$
M=\left(B_{0} \rtimes \Lambda_{0}\right)^{\bar{\otimes} \Gamma} \rtimes(\Gamma \rtimes \Gamma)=B_{0}^{\bar{\otimes} \Gamma} \rtimes\left(\Lambda_{0}^{(\Gamma)} \rtimes(\Gamma \times \Gamma)\right),
$$

where $R_{0}=B_{0} \rtimes \Lambda_{0}$ is a gms decomposition of $R_{0}$ that is globally invariant under $\beta$. We could not prove that all Cartan subalgebras of $M$ are unitarily conjugate to an obvious one, but we could prove that $M$ only has the obvious gms decompositions.

The above result leaves a complete freedom in the choice of $\mathbb{F}_{\infty} \curvearrowright^{\beta} R_{0}$. For specific choices of $\beta$, all $\beta$-invariant gms decompositions of $R_{0}$ can be determined and this leads to a proof of Theorem 3.7. All this entirely relies on the dual coaction (3.1) induced by another gms decomposition of $M$. To prove the same result for arbitrary Cartan subalgebras, a conceptually new technique has to be found.

3.7. Non commutative Bernoulli actions. The general dichotomy theorem of [PV11, PV12] discussed at the end of Section 3.5 can also be used to prove classification results for crossed product factors of type III. A particularly interesting class of type III factors arises from non commutative Bernoulli actions, see [Co74]. Whenever $\left(P_{0}, \varphi_{0}\right)$ is a factor equipped with a faithful normal state and $\Gamma$ is a countably infinite group, the Bernoulli action $\alpha$ of $\Gamma$ with base $\left(P_{0}, \varphi_{0}\right)$ is defined as follows. First denote by $(P, \varphi)=\bar{\otimes}_{g \in \Gamma}\left(P_{0}, \varphi_{0}\right)$ the infinite tensor product and by $\pi_{h}: P_{0} \rightarrow P$ the embedding as the $h$ 'th tensor factor. Then define the state preserving action $\Gamma \curvearrowright^{\alpha} P$ by $\alpha_{g}\left(\pi_{h}(x)\right)=\pi_{g h}(x)$ for all $g, h \in \Gamma, x \in P_{0}$. We still denote by $\varphi$ the natural extension of $\varphi$ to a faithful normal state on $M=P \rtimes \Gamma$.

In the entirely amenable case, when $P_{0}$ is an amenable factor, $P_{0} \neq \mathbb{C} 1$, and $\Gamma$ is an infinite amenable group, we have that $M=P \rtimes \Gamma$ is an amenable factor and is thus classified as follows by [Co75, Ha85]. Denote by $\left(\sigma_{t}^{\varphi_{0}}\right)_{t \in \mathbb{R}}$ the modular automorphism group of the faithful normal state $\varphi_{0}$, see Section 2.2. Then there are exactly three possibilities:

- if $\sigma_{t}^{\varphi_{0}}=\mathrm{id}$ for all $t \in \mathbb{R}$, then $\varphi_{0}$ is a tracial state and $M$ is the unique hyperfinite $\mathrm{II}_{1}$ factor $R$ (see Section 2.3 ),

- if $\sigma_{t}^{\varphi_{0}}$ is periodic with period $2 \pi /|\log \lambda|$ and $\lambda \in(0,1)$, then $M$ is the unique hyperfinite III $_{\lambda}$ factor $R_{\lambda}$ (see Section 2.4),

- if $\sigma_{t}^{\varphi_{0}}$ is not periodic, then $M$ is the unique hyperfinite $\mathrm{III}_{1}$ factor. 
When the group $\Gamma$ is non amenable, the classification of the crossed product factors $M=P \rtimes \Gamma$ changes drastically. We still assume that $P_{0}$ is a non trivial amenable factor and we also assume that the state $\varphi_{0}$ is almost periodic ${ }^{6}$. Then $M$ is an almost periodic full factor in the sense of [Co74] and the corresponding invariant $\operatorname{Sd}(M)$ of [Co74] equals the subgroup $\operatorname{Sd}\left(\varphi_{0}\right)$ of $\mathbb{R}_{+}^{*}$ generated by the eigenvalues of $\varphi_{0}$. This applies in particular to $P_{0}=M_{k}(\mathbb{C})$ and $\varphi_{0}=\operatorname{Tr}\left(\Delta_{0} \cdot\right)$ where $\Delta_{0} \in M_{k}(\mathbb{C})$ is a non singular, positive matrix of trace 1 . In that case, $\operatorname{Sd}\left(\varphi_{0}\right)$ equals the subgroup of $\mathbb{R}_{+}^{*}$ generated by the ratios between the eigenvalues of $\Delta_{0}$. In this way, we thus obtain many non isomorphic factors of type $\mathrm{III}_{1}$, distinguished by their Sd invariant.

When $\Gamma$ is a free group, the Sd invariant together with the group $\Gamma$ form a complete invariant in the following precise way.

Theorem 3.8 (V-Verraedt, [VV14]). The family of factors of the form

$$
\left\{\begin{array}{l|l}
\left(P_{0}, \varphi_{0}\right)^{\mathbb{F}_{n}} \rtimes \mathbb{F}_{n} \mid \begin{array}{l}
n \geq 2 \text { and }\left(P_{0}, \varphi_{0}\right) \text { is an amenable non trivial factor } \\
\text { with an almost periodic faithful normal state }
\end{array}
\end{array}\right\}
$$

is exactly classified, up to isomorphism, by the integer $n$ and the subgroup $\operatorname{Sd}\left(\varphi_{0}\right)$ of $\mathbb{R}_{+}^{*}$ generated by the eigenvalues of $\varphi_{0}$.

The main steps in the proof of Theorem 3.8 are the following. If two factors as in Theorem 3.8, associated to Bernoulli actions of $\mathbb{F}_{n}$ and $\mathbb{F}_{m}$, are isomorphic, they obviously must have the same Sd invariant. Using the dichotomy theorem of [PV11], we also prove that these Bernoulli actions of $\mathbb{F}_{n}$ and $\mathbb{F}_{m}$ are cocycle conjugate, which in particular means that $\mathbb{F}_{n} \cong \mathbb{F}_{m}$ and thus $n=m$. To prove the converse implication, i.e. the isomorphism of two Bernoulli crossed products of $\mathbb{F}_{n}$ having the same Sd invariant, we rely on Ocneanu's classification theorem up to cocycle conjugacy [Oc85] for outer actions of amenable groups (in this case, $\mathbb{Z} \times \mathrm{Sd}$ ) on the hyperfinite $\mathrm{II}_{\infty}$ factor.

3.8. Free Araki-Woods factors. A natural way to construct hyperfinite factors of type III is through the canonical anticommutation relations (CAR) and the quasi-free states. In [Sh96], Shlyakhtenko discovered a free probability analog of the CAR algebras and their quasi-free states, using Voiculescu's free Gaussian functor. More concretely, to any orthogonal representation $\left(U_{t}\right)_{t \in \mathbb{R}}$ of $\mathbb{R}$ on a real Hilbert space $\mathcal{H}_{\mathbb{R}}$ is associated the free Araki-Woods factor $\Gamma\left(\mathcal{H}_{\mathbb{R}}, U\right)^{\prime \prime}$ and the free quasi-free state $\varphi$.

This construction goes as follows. Denote by $\mathcal{H}=\mathcal{H}_{\mathbb{R}}+\mathrm{i} \mathcal{H}_{\mathbb{R}}$ the complexification of $\mathcal{H}_{\mathbb{R}}$ and by

$$
\mathcal{F}(\mathcal{H})=\mathbb{C} \Omega \oplus \bigoplus_{n=1}^{\infty}(\underbrace{\mathcal{H} \otimes \cdots \otimes \mathcal{H}}_{n \text {-fold }})
$$

\footnotetext{
${ }^{6}$ A faithful normal state $\varphi$ on a von Neumann algebra $M$ is called almost periodic if the eigenvectors of $\varphi$ span a weakly dense $*$-subalgebra of $M$. Here, $x \in M$ is called an eigenvector of $\varphi$ if there exists a $\lambda>0$ such that $\varphi(x y)=\lambda \varphi(y x)$ for all $y \in M$. This last condition is equivalent with the condition that $\sigma_{t}^{\varphi}(x)=\lambda^{\text {it }} x$ for all $t \in \mathbb{R}$.
} 
the full Fock space. Here, $\Omega$ is a unit vector, called the vacuum vector. For every $\xi \in \mathcal{H}$, denote by $\ell(\xi)$ the left creation operator defined by

$$
\ell(\xi) \Omega=\xi \quad \text { and } \quad \ell(\xi)\left(\xi_{1} \otimes \cdots \otimes \xi_{n}\right)=\xi \otimes \xi_{1} \otimes \cdots \otimes \xi_{n} .
$$

The complexification of $\left(U_{t}\right)_{t \in \mathbb{R}}$ is still denoted in the same way and is a unitary representation of $\mathbb{R}$ on $\mathcal{H}$. So we can define the non singular, positive, self adjoint operator $\Delta$ on $\mathcal{H}$ such that $\Delta^{\mathrm{i} t}=U_{t}$. Define the anti-unitary operator $J: \mathcal{H} \rightarrow \mathcal{H}$ given by $J(\xi+\mathrm{i} \eta)=\xi-\mathrm{i} \eta$ for all $\xi, \eta \in \mathcal{H}_{\mathbb{R}}$ and put $S=J \Delta^{1 / 2}$. Writing $\mathcal{K}_{\mathbb{R}}=\{\xi \in D(S) \mid S(\xi)=\xi\}$, we have found a new real Hilbert subspace $\mathcal{K}_{\mathbb{R}} \subset \mathcal{H}$ with the properties that $\mathcal{K}_{\mathbb{R}} \cap \mathrm{i} \mathcal{K}_{\mathbb{R}}=\{0\}$ and $\mathcal{K}_{\mathbb{R}}+\mathrm{i} \mathcal{K}_{\mathbb{R}} \subset \mathcal{H}$ is dense. Every such pair $\mathcal{K}_{\mathbb{R}} \subset \mathcal{H}$ arises in this way from an orthogonal representation.

Definition 3.9 (Shlyakhtenko, [Sh96]). The free Araki-Woods factor associated with $\left(\mathcal{H}_{\mathbb{R}}, U\right)$ is defined as

$$
\Gamma\left(\mathcal{H}_{\mathbb{R}}, U\right)^{\prime \prime}=\left\{\ell(\xi)+\ell(\xi)^{*} \mid \xi \in \mathcal{K}_{\mathbb{R}}\right\}^{\prime \prime}=\left\{\ell(\xi)+\ell(S(\xi))^{*} \mid \xi \in D(S)\right\}^{\prime \prime} .
$$

The vector state $\varphi(x)=\langle x \Omega, \Omega\rangle$ given by the vacuum vector $\Omega$ is a faithful normal state on $\Gamma\left(\mathcal{H}_{\mathbb{R}}, U\right)^{\prime \prime}$ called the free quasi-free state.

Except in the trivial case where $\mathcal{H}_{\mathbb{R}}$ is one dimensional and $U_{t}=\mathrm{id}$, the von Neumann algebra $M=\Gamma\left(\mathcal{H}_{\mathbb{R}}, U\right)^{\prime \prime}$ is indeed a factor. When $U_{t}=$ id for all $t \in \mathbb{R}$, we get that $M$ is a $\mathrm{II}_{1}$ factor that is isomorphic to the free group factor $L\left(\mathbb{F}_{\operatorname{dim}_{\mathbb{R}}\left(\mathcal{H}_{\mathbb{R}}\right)}\right)$. When $U$ is periodic with period $2 \pi /|\log \lambda|$ and $\lambda \in(0,1)$, then $M$ is a factor of type III $_{\lambda}$. In all other cases (and thus almost always), $M$ is a factor of type $\mathrm{III}_{1}$. Together with the observation that the functor $\Gamma(\cdot)^{\prime \prime}$ transforms direct sums of orthogonal representations into free products of von Neumann algebras (with respect to the free quasi-free states), we see that the free Araki-Woods factors can be viewed as type III analogs of the free group factors.

When $U$ is almost periodic, we denote by $\operatorname{Sd}(U)$ the subgroup of $\mathbb{R}_{+}^{*}$ generated by the eigenvalues of $U$, i.e. the positive real numbers $\lambda>0$ for which there exists a non zero vector $\xi \in \mathcal{H}$ satisfying $U_{t}(\xi)=\lambda^{i t} \xi$ for all $t \in \mathbb{R}$. When $U$ is almost periodic, the free quasi-free state is almost periodic as well and Connes' $\mathrm{Sd}$ invariant of $\Gamma\left(\mathcal{H}_{\mathbb{R}}, U\right)^{\prime \prime}$ (see [Co74]) equals $\operatorname{Sd}(U)$.

In [Sh96], Shlyakhtenko proved the fundamental result that the Sd invariant is a complete invariant for the almost periodic free Araki-Woods factors. As always, we say that two von Neumann algebras $M$ and $N$ are isomorphic if there exists a bijective *-isomorphism between $M$ and $N$. So even when $M$ and $N$ are equipped with canonical states (e.g. the free quasi-free states), we do not assume that isomorphisms are state preserving.

Theorem 3.10 (Shlyakhtenko, [Sh96]). The free Araki-Woods factors $\Gamma\left(\mathcal{H}_{\mathbb{R}}, U\right)^{\prime \prime}$ with $U$ almost periodic and non trivial are completely classified, up to isomorphism, by the subgroup $\operatorname{Sd}(U) \subset \mathbb{R}_{+}^{*}$.

In particular, for every $\lambda \in(0,1)$, there is a unique free Araki-Woods factor of type $\mathrm{III}_{\lambda}$, denoted as $T_{\lambda}$ and realized by any periodic orthogonal representation 
with period $2 \pi /|\log \lambda|$. The factor $T_{\lambda}$ is the free probability analog of the Powers factor $R_{\lambda}$ (see Section 2.4).

For non almost periodic representations $U$, the classification of the free ArakiWoods factors is an outstanding open problem. However, in the recent article [HSV16], we obtained the first such classification theorem for a quite large family of non almost periodic representations $U$.

To formulate this result, recall that orthogonal representations of $\mathbb{R}$ are fully classified by their spectral invariants. More concretely, let $\mu$ be a symmetric probability measure on the Borel sets of $\mathbb{R}$ and let $m: \mathbb{R} \rightarrow \mathbb{N} \cup\{+\infty\}$ be a symmetric Borel function (called the multiplicity function) that we always assume to satisfy $m(x) \geq 1$ for $\mu$-a.e. $x \in \mathbb{R}$. The orthogonal representation $\left(\mathcal{H}_{\mathbb{R}}, U\right)$ associated with $(\mu, m)$ is defined as follows. For all $n \in \mathbb{N} \cup\{+\infty\}$, define the symmetric Borel set $X_{n} \subset \mathbb{R}$ given by $X_{n}=\{x \in \mathbb{R} \mid m(x)=n\}$. Then define

$$
\begin{aligned}
& \mathcal{H}_{\mathbb{R}}=\bigoplus_{n \in \mathbb{N} \cup\{+\infty\}} \bigoplus_{k=1}^{n} \mathcal{H}_{\mathbb{R}}^{n} \\
& \text { with } \quad \mathcal{H}_{\mathbb{R}}^{n}=\left\{\xi \in L^{2}\left(X_{n}, \mu\right) \mid \forall x \in X_{n}: \xi(-x)=\overline{\xi(x)}\right\}, \\
& \text { and }\left(U_{t} \xi\right)(x)=\exp (\mathrm{i} t x) \xi(x) .
\end{aligned}
$$

Every orthogonal representation of $\mathbb{R}$ on a separable real Hilbert space is orthogonally equivalent with the representation associated with a pair $(\mu, m)$ as above. And the representations associated with $\left(\mu_{i}, m_{i}\right), i=1,2$, are orthogonally equivalent if and only if $\mu_{1} \sim \mu_{2}$ (meaning that $\mu_{1}$ and $\mu_{2}$ belong to the same measure class, i.e. have the same Borel sets of measure zero) and $m_{1}(x)=m_{2}(x)$ for $\mu_{i}$-a.e. $x \in \mathbb{R}$. For all $(\mu, m)$, we denote by $\Gamma(\mu, m)^{\prime \prime}$ the free Araki-Woods factor associated with the above orthogonal representation of $\mathbb{R}$. Every free Araki-Woods factor arises in this way.

Observe that the almost periodic case corresponds to the case where $\mu$ is an atomic measure. For an arbitrary Borel measure $\mu$ on $\mathbb{R}$, we denote by $\mu=\mu_{a}+\mu_{c}$ its unique decomposition as the sum of an atomic and a continuous measure. We denote by $\Lambda\left(\mu_{a}\right) \subset \mathbb{R}$ the subgroup of $(\mathbb{R},+)$ generated by the atoms of $\mu_{a}$. So, when $\mu$ is atomic, we get that $\operatorname{Sd}(U)=\exp (\Lambda(\mu))$ and Theorem 3.10 can be reinterpreted as saying that the free Araki-Woods factors $\Gamma(\mu, m)^{\prime \prime}$ with $\mu$ atomic are completely classified by the subgroup $\Lambda(\mu) \subset \mathbb{R}$. In particular, they do not depend on the multiplicity function $m$.

In [HSV16], we consider the class $\mathcal{S}(\mathbb{R})$ of symmetric probability measures $\mu$ satisfying the following two properties: $\mu$ has at least one atom different from 0 and $\mu_{c} * \mu_{c} \prec \mu_{c}$, meaning that the convolution product $\mu_{c} * \mu_{c}$ is absolutely continuous w.r.t. $\mu_{c}$. We then prove the following result.

Theorem 3.11 (Houdayer-Shlyakhtenko-V, [HSV16]). The free Araki-Woods factors $\Gamma(\mu, m)^{\prime \prime}$ with $\mu \in \mathcal{S}(\mathbb{R})$ are completely classified by the subgroup $\Lambda\left(\mu_{a}\right) \subset \mathbb{R}$ and the measure class of $\mu_{c} * \delta_{\Lambda\left(\mu_{a}\right)}$, where $\delta_{\Lambda\left(\mu_{a}\right)}$ denotes any atomic probability measure whose set of atoms equals $\Lambda\left(\mu_{a}\right)$.

The key step to prove Theorem 3.11 is the following: whenever $\Gamma(\mu, m)^{\prime \prime} \cong$ $\Gamma(\nu, n)^{\prime \prime}$ and $\nu$ has at least one atom different from 0 , then there also exists an 
isomorphism preserving the free quasi-free states. That result is in turn based on the following intrinsic characterization of the free quasi-free state proved in [HSV16]: up to conjugacy by a partial isometry and "taking corners", the free quasi-free state $\varphi$ on an arbitrary free Araki-Woods factor $M=\Gamma(\mu, m)^{\prime \prime}$ with $\mu$ having at least one atom different from 0 is the only faithful normal state $\psi$ whose centralizer ${ }^{7} M^{\psi}$ is non amenable.

So again, we have obtained a classification theorem for von Neumann algebras that is based on the dichotomy between amenability and non amenability. This connects very well with another wide open problem on type III factors posed by Connes [Co80]: does every type III $_{1}$ factor $M$ (acting on a separable Hilbert space) have a trivial bicentralizer ${ }^{8}$ ? By [Ha85], this problem is equivalent to the question whether $M$ admits a faithful normal state $\psi$ such that $\left(M^{\psi}\right)^{\prime} \cap M=\mathbb{C} 1$.

Another result of [HSV16], along the same lines as the above mentioned characterization of the free quasi-free state, says that if $\mu$ is a continuous measure, then all faithful normal states $\psi$ on $M=\Gamma(\mu, m)^{\prime \prime}$ have an amenable centralizer $M^{\psi}$. This is an optimal result: although the centralizer of the free quasi-free state equals $\mathbb{C} 1$, by [Ho08], $M$ satisfies Connes' bicentralizer problem and thus admits a faithful normal state $\psi$ such that $M^{\psi}$ is an irreducible subfactor of $M$.

\section{References}

[BV14] M. Berbec and S. Vaes, W*-superrigidity for group von Neumann algebras of left-right wreath products. Proc. Lond. Math. Soc. 108 (2014), 1113-1152.

[CS11] I. Chifan and T. Sinclair, On the structural theory of $\mathrm{II}_{1}$ factors of negatively curved groups. Ann. Sci. École Norm. Sup. 46 (2013), 1-34.

[Co72] A. Connes, Une classification des facteurs de type III. Ann. Sci. École Norm. Sup. 6 (1973), 133-252.

[Co74] A. Connes, Almost periodic states and factors of type $\mathrm{III}_{1}$. J. Funct. Anal. 16 (1974), 415-445.

[Co75] A. Connes, Classification of injective factors. Ann. of Math. 104 (1976), 73-115.

[Co80] A. Connes, Classification des facteurs. In Operator algebras and applications, Part 2 (Kingston, 1980), Proc. Sympos. Pure Math. 38, Amer. Math. Soc., Providence, 1982, pp. 43-109.

[FM75] J. Feldman and C.C. Moore, Ergodic equivalence relations, cohomology, and von Neumann algebras. II. Trans. Amer. Math. Soc. 234 (1977), 325-359.

\footnotetext{
${ }^{7}$ The centralizer $M^{\psi}$ of a faithful normal state $\psi$ on a von Neumann algebra $M$ is defined as the von Neumann subalgebra of all $x \in M$ such that $\psi(x y)=\psi(y x)$ for all $y \in M$. Then, $M^{\psi}=\left\{x \in M \mid \sigma_{t}^{\psi}(x)=x\right.$ for all $\left.t \in \mathbb{R}\right\}$.

${ }^{8}$ This problem is also strongly related to the uniqueness of the amenable type $\mathrm{III}_{1}$ factor. Connes proved in [Co75] that every amenable factor is hyperfinite. He also proved that all hyperfinite III $_{1}$ factors with a trivial bicentralizer are isomorphic. Haagerup proved in [Ha85] that all amenable III $_{1}$ factors have a trivial bicentralizer. Whether the same holds for arbitrary $\mathrm{III}_{1}$ factors (acting on a separable Hilbert space) is not known.
} 
[Fu11] A. Furman, A survey of measured group theory. In Geometry, rigidity, and group actions. Chicago Lectures in Math., Univ. Chicago Press, Chicago, 2011, pp. 296-374.

[Ga01] D. Gaboriau, Invariants $\ell^{2}$ de relations d'équivalence et de groupes. Publ. Math. Inst. Hautes Études Sci. 95 (2002), 93-150.

[Ga10] D. Gaboriau, Orbit equivalence and measured group theory. In Proceedings of the International Congress of Mathematicians (Hyderabad, 2010), Vol. III, Hindustan Book Agency, New Delhi, 2010, pp. 1501-1527.

[Ha85] U. Haagerup, Connes' bicentralizer problem and uniqueness of the injective factor of type $\mathrm{III}_{1}$. Acta Math. 158 (1987), 95-148.

[Ho08] C. Houdayer, Free Araki-Woods factors and Connes' bicentralizer problem. Proc. Amer. Math. Soc. 137 (2009), 3749-3755.

[HSV16] C. Houdayer, D. Shlyakhtenko and S. Vaes, Classification of a family of non almost periodic free Araki-Woods factors. Preprint. arXiv:1605.06057

[Io10] A. Ioana, $\mathrm{W}^{*}$-superrigidity for Bernoulli actions of property (T) groups. $J$. Amer. Math. Soc. 24 (2011), 1175-1226.

[Io12a] A. Ioana, Classification and rigidity for von Neumann algebras. In European Congress of Mathematics (Krakow, 2012), European Mathematical Society (EMS), Zürich, 2013, pp. 601-625.

[Io12b] A. Ioana, Cartan subalgebras of amalgamated free product $\mathrm{II}_{1}$ factors. Ann. Sci. École Norm. Sup. 48 (2015), 71-130.

[IPV10] A. Ioana, S. Popa and S. Vaes, A class of superrigid group von Neumann algebras. Ann. Math. 178 (2013), 231-286.

[Ka67] R.V. Kadison, Problems on von Neumann algebras. Baton Rouge Conference on Operator Algebras, Louisiana State University, March 1967, unpublished.

[KV15] A.S. Krogager and S. Vaes, A class of $\mathrm{II}_{1}$ factors with exactly two crossed product decompositions. J. Math. Pures Appl., to appear. arXiv:1512.06677

[Mc69] D. McDuff, Uncountably many II 1 factors. Ann. of Math. 90 (1969), 372-377.

[MvN36] F.J. Murray and J. von Neumann, On rings of operators. Ann. of Math. 37 (1936), 116-229.

[MvN43] F.J. Murray and J. von Neumann, On rings of operators. IV. Ann. of Math. 44 (1943), 716-808.

[Oc85] A. Ocneanu, Actions of discrete amenable groups on von Neumann algebras. Lecture Notes in Mathematics 1138, Springer-Verlag, Berlin, 1985.

[OP07] N. Ozawa and S. Popa, On a class of $\mathrm{II}_{1}$ factors with at most one Cartan subalgebra I. Ann. Math. 172 (2010), 713-749.

[Pe09] J. Peterson, Examples of group actions which are virtually $\mathrm{W}^{*}$-superrigid. Preprint. arXiv: 1002.1745

[Po01] S. Popa, On a class of type $\mathrm{II}_{1}$ factors with Betti numbers invariants. Ann. of Math. 163 (2006), 809-899.

[Po03] S. Popa, Strong rigidity of $\mathrm{II}_{1}$ factors arising from malleable actions of $w$-rigid groups, I. Invent. Math. 165 (2006), 369-408. 
[Po04] S. Popa, Strong rigidity of $\mathrm{II}_{1}$ factors arising from malleable actions of $w$-rigid groups, II. Invent. Math. 165 (2006), 409-452.

[Po05] S. Popa, Cocycle and orbit equivalence superrigidity for malleable actions of w-rigid groups. Invent. Math. 170 (2007), 243-295.

[Po06] S. Popa, Deformation and rigidity for group actions and von Neumann algebras. In Proceedings of the International Congress of Mathematicians (Madrid, 2006), Vol. I, European Mathematical Society Publishing House, 2007, pp. 445-477.

[PV09] S. Popa and S. Vaes, Group measure space decomposition of $\mathrm{II}_{1}$ factors and W*-superrigidity. Invent. Math. 182 (2010), 371-417.

[PV11] S. Popa and S. Vaes, Unique Cartan decomposition for $\mathrm{II}_{1}$ factors arising from arbitrary actions of free groups. Acta Math. 212 (2014), 141-198.

[PV12] S. Popa and S. Vaes, Unique Cartan decomposition for $\mathrm{II}_{1}$ factors arising from arbitrary actions of hyperbolic groups. J. Reine Angew. Math. 694 (2014), 215239 .

[Sh96] D. Shlyakhtenko, Free quasi-free states. Pacific J. Math. 177 (1997), 329-368.

[Ta70] M. Takesaki, Tomita's theory of modular Hilbert algebras and its applications. Lecture Notes in Mathematics 128, Springer-Verlag, Berlin, New York, 1970.

[Ta73] M. Takesaki, Duality for crossed products and the structure of von Neumann algebras of type III. Acta Math. 131 (1973), 249-310.

[Va10] S. Vaes, Rigidity for von Neumann algebras and their invariants. In Proceedings of the International Congress of Mathematicians (Hyderabad, 2010), Vol. III, Hindustan Book Agency, New Delhi, 2010, pp. 1624-1650.

[VV14] S. Vaes and P. Verraedt, Classification of type III Bernoulli crossed products. Adv. Math. 281 (2015), 296-332.

[vN29] J. von Neumann, Zur allgemeinen Theorie des Masses. Fund. Math. 13 (1929), 73-116.

Stefaan Vaes, KU Leuven, Department of Mathematics, Celestijnenlaan 200B box 2400, B-3001 Leuven, Belgium

E-mail: stefaan.vaes@kuleuven.be 